\title{
Contralateral interoptic approach to paraclinoid aneurysms: a patient-selection algorithm based on anatomical investigation and clinical validation
}

\author{
Xiaochun Zhao, MD, Ali Tayebi Meybodi, MD, Mohamed A. Labib, MD, CM, Sirin Gandhi, MD, \\ Evgenii Belykh, MD, PhD, Komal Naeem, MD, Mark C. Preul, MD, Peter Nakaji, MD, and \\ Michael T. Lawton, MD
}

Department of Neurosurgery, Barrow Neurological Institute, St. Joseph's Hospital and Medical Center, Phoenix, Arizona

\begin{abstract}
OBJECTIVE Aneurysms that arise on the medial surface of the paraclinoid segment of the internal carotid artery (ICA) are surgically challenging. The contralateral interoptic trajectory, which uses the space between the optic nerves, can partially expose the medial surface of the paraclinoid ICA. In this study, the authors quantitatively measure the area of the medial ICA accessible through the interoptic triangle and propose a potential patient-selection algorithm that is based on preoperative measurements on angiographic imaging.

METHODS The contralateral interoptic trajectory was studied on 10 sides of 5 cadaveric heads, through which the medial paraclinoid ICA was identified. The falciform ligament medial to the contralateral optic canal was incised, the contralateral optic nerve was gently elevated, and the medial surface of the paraclinoid ICA was inspected via different viewing angles to obtain maximal exposure. The accessible area on the carotid artery was outlined. The distance from the distal dural ring (DDR) to the proximal and distal borders of this accessible area was measured. The superior and inferior borders were measured using the clockface method relative to a vertical line on the coronal plane. To validate these parameters, preoperative measurements and intraoperative findings were reviewed in 8 clinical cases.
\end{abstract}

RESULTS In the sagittal plane, the mean (SD) distances from the DDR to the proximal and distal ends of the accessible area on the paraclinoid ICA were $2.5(1.52) \mathrm{mm}$ and $8.4(2.32) \mathrm{mm}$, respectively. In the coronal plane, the mean (SD) angles of the superior and inferior ends of the accessible area relative to a vertical line were $21.7^{\circ}\left(14.84^{\circ}\right)$ and $130.9^{\circ}$ $\left(12.75^{\circ}\right)$, respectively. Six $(75 \%)$ of 8 clinical cases were consistent with the proposed patient-selection algorithm.

CONCLUSIONS The contralateral interoptic approach is a feasible route to access aneurysms that arise from the medial paraclinoid ICA. An aneurysm can be safely clipped via the contralateral interoptic trajectory if 1) both proximal and distal borders of the aneurysm neck are $2.5-8.4 \mathrm{~mm}$ distal to the DDR, and 2 ) at least one border of the aneurysm neck on the coronal clockface is $21.7^{\circ}-130.9^{\circ}$ medial to the vertical line.

https://thejns.org/doi/abs/10.3171/2020.3.JNS193205

KEYWORDS contralateral approach; paraclinoid aneurysms; vascular disorders

A CCESSING aneurysms that arise on the medial wall of the paraclinoid internal carotid artery (ICA) through the interoptic triangle (IOT) is surgically challenging because this trajectory is relatively long and narrow and because of the contiguity of various vital neurovascular structures (Fig. 1).,2 Despite these challenges, this approach can be straightforward and effective with appropriate patient selection (i.e., patients with small aneurysms that point medially and superiorly) since this approach offers direct access to the aneurysm without anterior clinoidectomy. ${ }^{2}$ In addition, in patients with multiple aneurysms in whom the more formidable aneurysm is lo- cated on the side opposite the paraclinoid aneurysm, the best management may be ipsilateral clipping of the more difficult-to-treat aneurysm and contralateral clipping of the paraclinoid aneurysm. The anatomical relationships between the neurovascular structures, the accessibility of this trajectory, and other related factors have been thoroughly investigated in the literature. ${ }^{3-6}$ However, to our knowledge, no preoperative algorithm has been developed for approach selection, which could help in deciding whether this challenging but effective approach should be selected for the treatment of certain aneurysms. We conducted a quantitative anatomical study to develop a pre-

ABBREVIATIONS DDR = distal dural ring; ICA = internal carotid artery; IOT = interoptic triangle.

SUBMITTED November 26, 2019. ACCEPTED March 13, 2020.

INCLUDE WHEN CITING Published online June 12, 2020; DOI: 10.3171/2020.3.JNS193205. 

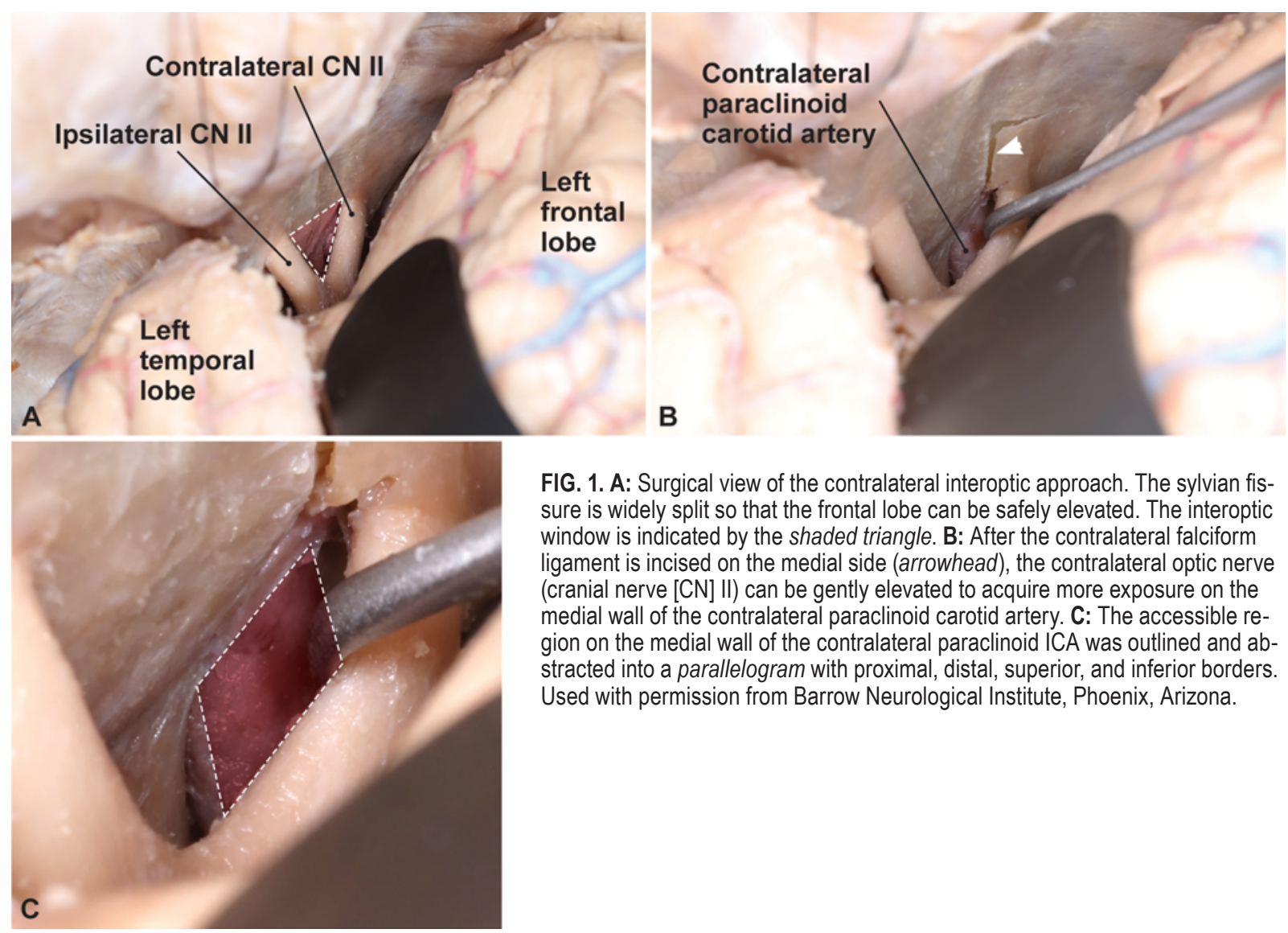

FIG. 1. A: Surgical view of the contralateral interoptic approach. The sylvian fissure is widely split so that the frontal lobe can be safely elevated. The interoptic window is indicated by the shaded triangle. B: After the contralateral falciform ligament is incised on the medial side (arrowhead), the contralateral optic nerve (cranial nerve [CN] II) can be gently elevated to acquire more exposure on the medial wall of the contralateral paraclinoid carotid artery. C: The accessible region on the medial wall of the contralateral paraclinoid ICA was outlined and abstracted into a parallelogram with proximal, distal, superior, and inferior borders. Used with permission from Barrow Neurological Institute, Phoenix, Arizona.

operative CTA-based algorithm for decision-making and subsequently validated this algorithm with clinical cases that used this approach.

\section{Methods}

Given the retrospective and anonymous nature of the data collection in this study, institutional review board approval was not required.

\section{Anatomical Investigation}

\section{Surgical Approach}

Ten sides of 5 cadaveric heads embalmed in an alcohol-based solution with arteries and veins injected with red- and blue-colored silicone, respectively, were used in the present study. Because the accessibility of the medial paraclinoid ICA is largely dependent on the size of the IOT, 3,7 neither orbit roof removal nor anterior clinoidectomy was necessary. ${ }^{4} \mathrm{~A}$ standard pterional craniotomy was used, as in most published case series., ${ }^{2,8,9}$

During the intracranial dissection, the sylvian fissure was fully split to maximize the frontal lobe elevation, and the carotid cistern was entered by sharply opening the arachnoid membrane around the optic nerve and carotid artery. A subfrontal approach was established after subsequent arachnoid dissection between the basal frontal lobe and the optic nerves. Meticulous care was taken to prevent damage to the ipsilateral olfactory nerve during explora- tion. The IOT can be identified after the frontal lobe is sufficiently separated from the optic apparatus. ${ }^{9}$

In the literature, contralateral falciform ligament disconnection $^{5}$ (Fig. 1B) and tuberculum sellae drilling ${ }^{10}$ are the two methods that have been reported to significantly expand the size of the IOT and the exposure of the contralateral paraclinoid ICA.,7 Because drilling the contralateral portion of the tuberculum sellae is relatively invasive and is associated with a high risk of entering the sphenoid or ethmoid sinus and causing cerebrospinal fluid leakage, ${ }^{4}$ we performed only a contralateral falciform ligament disconnection in this study.

The contralateral optic nerve was gently elevated to increase exposure while minimizing the risk of damage to the nerve. The medial surface of the contralateral paraclinoid ICA was exposed, and the exposed area was outlined with methylene blue dye. Multiple viewing angles were assessed by moving the microscope around to maximize the exposable (outlined) area through the IOT. This outlined area was considered as a parallelogram on the medial wall of the contralateral paraclinoid ICA, which included proximal, distal, superior, and inferior borders as its four sides (Fig. 1C).

\section{Quantitative Measurements}

Distances between the distal dural ring (DDR) and the proximal and distal borders of the outlined area were measured, and a line connecting the superior edge of the optic 
strut and lateral edge of the tuberculum sellae was considered to be an approximation of the DDR (Fig. 2). The coronal cross-section of the paraclinoid ICA was abstracted as a clockface with an imaginary vertical line passing though the center. The superior and inferior borders on the coronal section were considered as 2 points on the circle. The superior and inferior borders were reported as angles on the clockface: the 12 o'clock point was marked as $0^{\circ}$, a point on the medial half-circle was reported as a positive degree $\left(0^{\circ}\right.$ to $\left.180^{\circ}\right)$, and a point on the lateral half-circle was reported as a negative degree $\left(0^{\circ}\right.$ to $\left.-180^{\circ}\right)$.

In summary, the mean distances from the DDR to the proximal and distal borders of the accessible area were measured, indicating the approximate length of the accessible area and distance to the DDR in the sagittal plane. The angles of the superior and inferior borders of the accessible area were measured, indicating the approximate width of the accessible angle in the coronal plane. In addition, the distance between bilateral optic nerves at the entrance of the optic canal and the length from the anterior aspect of the optic chiasm to the limbus sphenoidale were measured to evaluate the size of the IOT. ${ }^{3}$

The measurements were performed using a navigation system (StealthStation Image-guidance Workstation, Medtronic Surgical Navigation Technologies). Every point was regarded as a spatial point with 3 coordinates (i.e., $x$, $\mathrm{y}$, and $\mathrm{z}$; units were millimeters). The distance between 2 points was calculated using the Pythagorean theorem. Angles on the clockface were measured with a protractor after the carotid segment was removed from the cadaver.

\section{Clinical Validation}

Eight cases of paraclinoid aneurysms that had been treated via the IOT by the senior author (M.T.L.) between 2008 and 2019 were reviewed. The distances between the proximal and distal sides of the aneurysm neck to the DDR were measured in the sagittal plane, and the angles of the superior and inferior borders of the aneurysm neck were measured in the coronal plane using the clockface method on preoperative CTA or angiographic images.

The intraoperative exposure to the aneurysm was evaluated by reviewing the surgical videos. Whether the proximal, distal, superior, and inferior sides of the aneurysm neck could be accessed was evaluated. Finally, correlation and validation of the proposed algorithm were performed on the basis of the preoperative measurements and the intraoperative findings.

\section{Statistical Analysis}

The statistical analysis was performed using PASW Statistics version 18.0.0 (IBM Corp.). Data are reported as means (standard deviations).

\section{Results}

\section{Anatomical Investigation}

In the sagittal plane, the segment of the paraclinoid ICA accessible through the IOT was located within a mean of 2.5 (1.52) to $8.4(2.32) \mathrm{mm}$ distal to the DDR. In the coronal plane, the IOT provided access to the area of $21.7^{\circ}$ $\left(14.84^{\circ}\right)$ to $130.9^{\circ}\left(12.75^{\circ}\right)$ on the medial surface of the

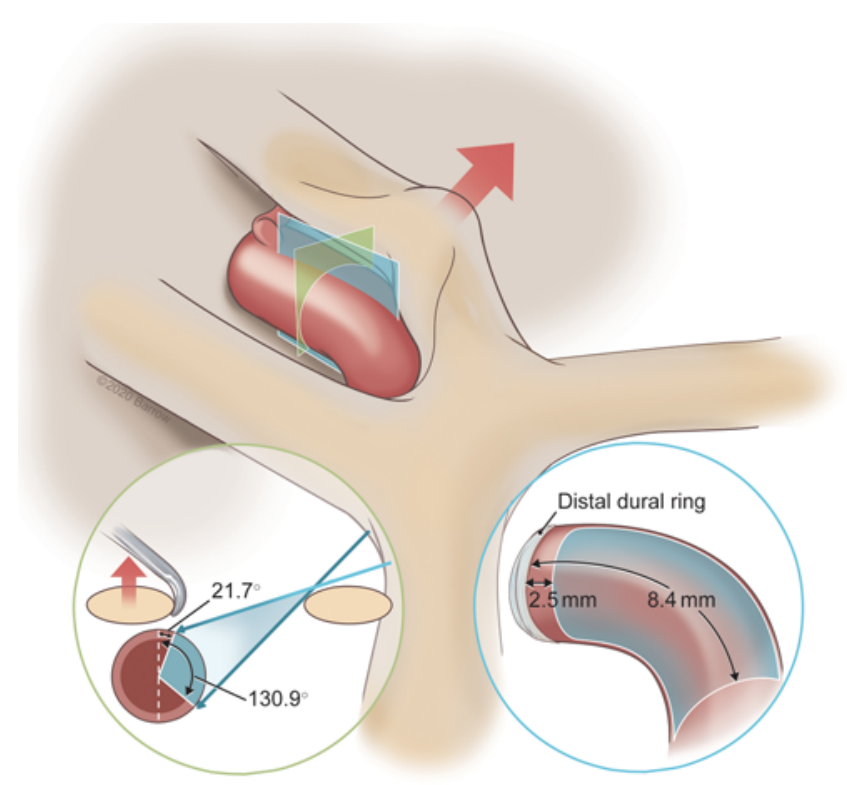

FIG. 2. Illustration of the surgical view and methods of measurement. The mean distances from the DDR to the proximal and distal accessible areas were measured, representing the accessible range of length in the sagittal plane (blue). Angles from the vertical line to the superior and inferior accessible areas were measured, representing the accessible range of angle in the coronal clockface (green). Used with permission from Barrow Neurological Institute, Phoenix, Arizona.

paraclinoid ICA (Fig. 2). The mean exposed paraclinoid ICA segment was 6.0 (1.29) $\mathrm{mm}$ long.

The mean area of the IOT was $66.3(37.96) \mathrm{mm}^{2}$, the mean distance between bilateral optic nerves was 13.7 (2.48) $\mathrm{mm}$, and the mean distance from the anterior aspect of the optic chiasm to the limbus sphenoidale was 9.3 (3.21) $\mathrm{mm}$.

\section{Patient-Selection Algorithm}

The direction of the IOT approach is perpendicular to the course of the paraclinoid ICA and parallel to the coronal plane (Fig. 3). In the sagittal plane, movement of the clip tip in a proximal to distal direction along the ICA is limited by the tuberculum sellae anteriorly and the optic chiasm posteriorly; therefore, it is imperative to expose the aneurysm neck both proximally and distally. In the coronal plane, in contrast, moving the clip tip around the caliber of the ICA is feasible (either sliding the clip on top of the ICA to the contralateral side or sliding the clip tip inferiorly medial to the ICA within the IOT; Fig. 3A and B). Therefore, complete aneurysm ligation can be achieved even when one side of the aneurysm neck, either superior or inferior, is hidden from direct visualization via the IOT. However, complete exposure of the neck both superiorly and inferiorly is preferable (Fig. 3C).

On the basis of the quantitative measurements, we propose the following method for preoperative patient selection. A paraclinoid aneurysm can be safely clipped via the contralateral interoptic trajectory if 1) both sides of the aneurysm neck in the proximal-distal dimension are 2.5-8.4 mm distal to the DDR and 2) at least one side of 

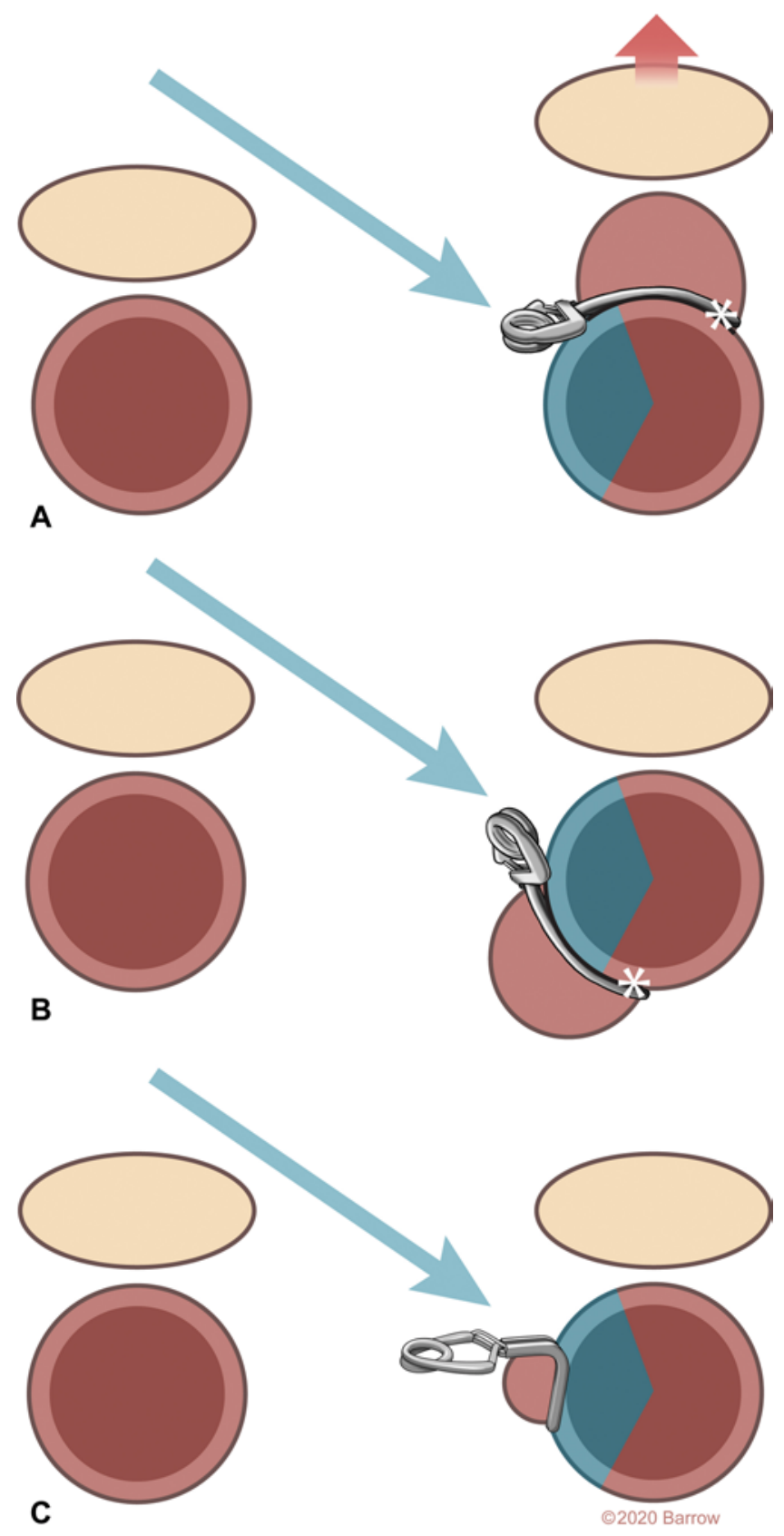

FIG. 3. Schematic illustration of three locations of accessible paraclinoid aneurysms in the coronal plane. A: A left ophthalmic artery aneurysm that points upward is demonstrated. The mass effect of the aneurysm elevates the optic nerve, and although the superior side of the neck is not exposed (white asterisk), clip application is still feasible. B: A left superior hypophyseal artery aneurysm that points medially and inferiorly is demonstrated. Although the inferior side of the neck is not exposed (white asterisk), clip application is still feasible. C: An aneurysm on the medial wall of the left paraclinoid carotid artery is demonstrated. Both the superior and the inferior sides of the neck can be exposed, which is the most favorable configuration for a contralateral interoptic approach. Ovals represent optic nerves; red circles, carotid arteries; irregular red bulges, aneurysms; blue arrows, the contralateral interoptic trajectory; and blue shaded sectors, the exposable area. Used with permission from Barrow Neurological Institute, Phoenix, Arizona. the aneurysm neck on the coronal clockface is within the range of $21.7^{\circ}-130.9^{\circ}$ medial to the vertical line.

\section{Clinical Validation and Illustrative Cases}

Preoperative measurements and intraoperative findings are reported in Fig. 4 and Table 1. Six (75\%) of 8 cases matched the proposed method when the preoperative measurements were correlated with the intraoperative findings. The 2 cases that were not compatible with the method were case 3 (Fig. 4C) and case 5 (Fig. 4E). In case 3 , an ophthalmic artery aneurysm was located underneath the optic nerve and pointed superiorly. The superior border and inferior border of the aneurysm neck were $30^{\circ}$ on the lateral side (presented as $-30^{\circ}$ ) and $5^{\circ}$ medially (presented as $5^{\circ}$ ). Also, the measurement of the proximal aneurysm neck $(2.3 \mathrm{~mm})$ was $0.2 \mathrm{~mm}$ less than the lower boundary of the range specified in our method $(2.5 \mathrm{~mm})$. In the operation, the contralateral optic nerve was substantially elevated by a suction tip to better expose the aneurysm. In this way, the superiorly pointing aneurysm was eventually clipped; however, the lateral side of the aneurysm was not identified. In case 5 , the proximal aneurysm neck was $1.8 \mathrm{~mm}$ from the DDR, which was $0.7 \mathrm{~mm}$ more proximal than the lower boundary of the range specified in our method $(2.5 \mathrm{~mm})$. The relatively small size of the aneurysm $(1.3 \mathrm{~mm})$ permitted successful clipping in the narrow area between the optic nerve and tuberculum sellae. An illustrative case demonstrates application of the algorithm in Fig. 5 .

\section{Discussion}

We conducted a quantitative anatomical analysis with clinical validation to propose a potential method to help decide whether a paraclinoid aneurysm should be operated on via a contralateral IOT approach on the basis of preoperative imaging measurements.

\section{Necessity of Open Surgery in Paraclinoid Aneurysms}

Although endovascular therapies have been gradually replacing open operations, they have some limitations in certain circumstances. The rate of complete occlusion of an aneurysm can be low for complex vessel or aneurysm configurations. ${ }^{11}$ Moreover, small perforating branches may not be visible on the angiogram, and the sacrifice of these branches may cause ischemic complications. ${ }^{12-14}$ Furthermore, endovascular treatment does not relieve the mass effect on the optic nerve caused by the aneurysm.

In patients with paraclinoid aneurysms, minor but important arteries coming off the ICA (e.g., superior hypophyseal artery) can be inconspicuous on angiography. Characteristics of some aneurysms, such as wide-neck and superiorly pointing aneurysms (i.e., "true" ophthalmic aneurysms ${ }^{15}$ ) with mass effect to the optic nerve, do not favor endovascular treatments; in such cases, open surgery has advantages.

\section{Advantages of the Contralateral Interoptic Approach}

The contralateral interoptic trajectory is a narrow but straightforward approach to the medial surface of the 

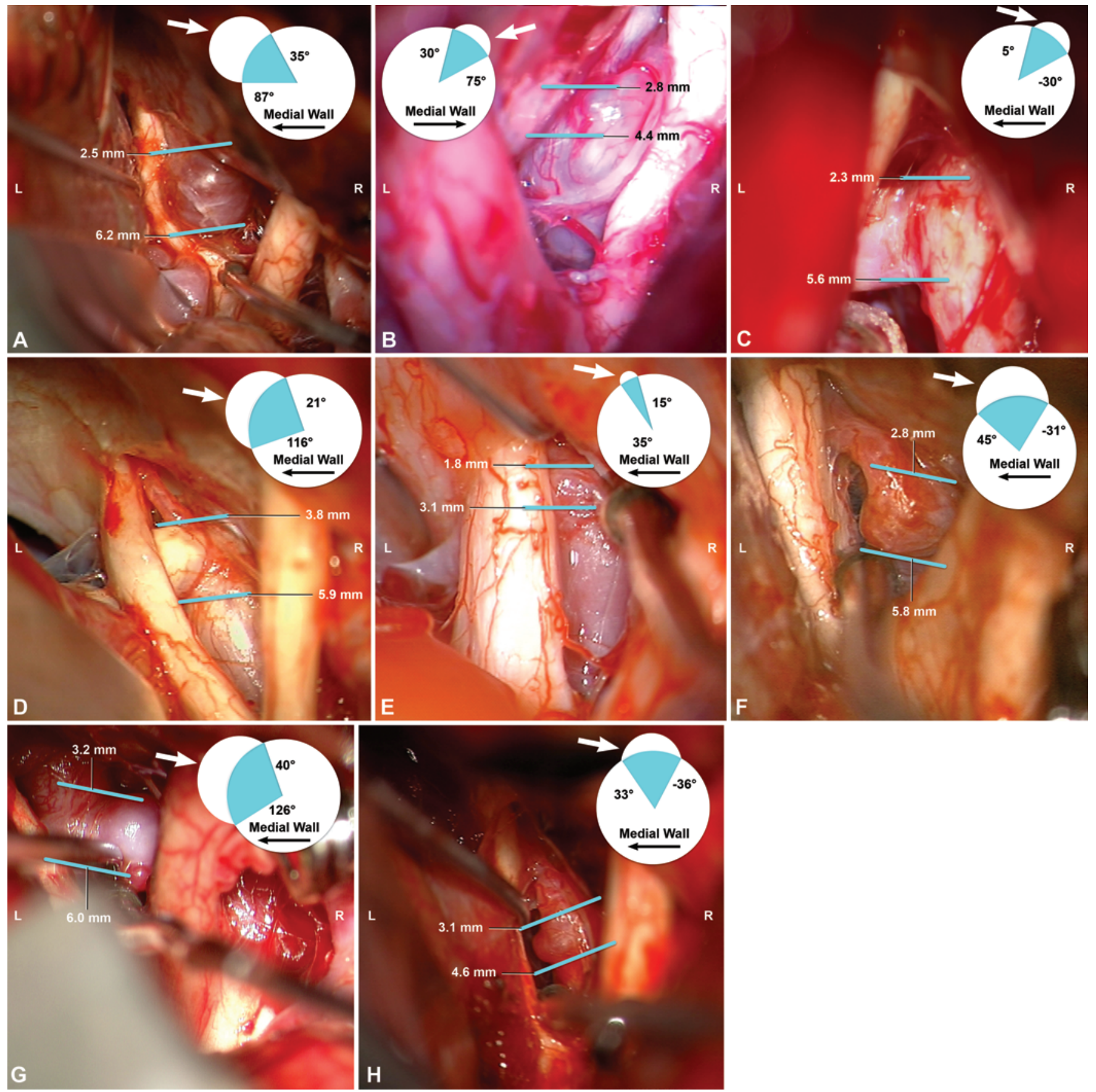

FIG. 4. Clinical validation by correlating the preoperative measurements and intraoperative findings using 8 case examples. The schematic illustrations indicate the pattern of the contralateral aneurysm on a coronal angiogram. A: Case 1. A 54-year-old woman underwent a right pterional craniotomy for a left ophthalmic segment artery (OSA) aneurysm. The aneurysm neck ranged from 2.5 to $6.2 \mathrm{~mm}$ in the sagittal plane and from $35^{\circ}$ to $87^{\circ}$ on the coronal clockface. This case matched the algorithm. B: Case 2. A 72-year-old woman underwent a left modified orbitozygomatic craniotomy for a right OSA aneurysm. The aneurysm neck ranged from 2.8 to $4.4 \mathrm{~mm}$ in the sagittal plane and from $30^{\circ}$ to $75^{\circ}$ on the coronal clockface. This case matched the algorithm. C: Case 3. A 53-year-old man underwent a right pterional craniotomy for a left OSA aneurysm. The aneurysm neck ranged from 2.3 to $5.6 \mathrm{~mm}$ in the sagittal plane and from $-30^{\circ}$ to $5^{\circ}$ on the coronal clockface. This case did not match the algorithm. The contralateral optic nerve was substantially elevated by the tip of the sucker (lower left corner). D: Case 4. A 79-year-old woman underwent a right pterional craniotomy for a left OSA aneurysm. The aneurysm neck ranged from 3.8 to $5.9 \mathrm{~mm}$ in the sagittal plane and from $21^{\circ}$ to $116^{\circ}$ on the coronal clockface. This case matched the algorithm. E: Case 5. A 79-year-old woman underwent a right pterional craniotomy for a left OSA aneurysm. The aneurysm neck ranged from 1.8 to $3.1 \mathrm{~mm}$ in the sagittal plane and from $15^{\circ}$ to $35^{\circ}$ on the coronal clockface. This case did not match the algorithm. F: Case 6. A 23-year-old man underwent a right pterional craniotomy for a left OSA aneurysm. The aneurysm neck ranged from 2.8 to $5.8 \mathrm{~mm}$ in the sagittal plane and from $-31^{\circ}$ to $45^{\circ}$ on the coronal clockface. This case matched the algorithm. G: Case 7 . A 39-year-old woman underwent a right pterional craniotomy for a left superior hypophyseal artery aneurysm. The aneurysm neck ranged from 3.2 to $6.0 \mathrm{~mm}$ in the sagittal plane and from $40^{\circ}$ to $126^{\circ}$ on the coronal clockface. This case matched the algorithm. H: Case 8. A 57-year-old woman underwent a right pterional craniotomy for a left OSA aneurysm. The aneurysm neck ranged from 3.1 to $4.6 \mathrm{~mm}$ in the sagittal plane and from $-36^{\circ}$ to $33^{\circ}$ on the coronal clockface. This case matched the algorithm. Published in accordance with the University of California statement on fair use. 
TABLE 1. Clinical examples for algorithm validation

\begin{tabular}{|c|c|c|c|c|c|c|c|c|c|}
\hline \multirow[b]{2}{*}{$\begin{array}{l}\text { Case } \\
\text { No. }\end{array}$} & \multirow[b]{2}{*}{$\begin{array}{l}\text { Age } \\
\text { (yrs) }\end{array}$} & \multirow[b]{2}{*}{ Sex } & \multirow[b]{2}{*}{ Brief Case Presentation } & \multicolumn{2}{|c|}{ Distance (mm) } & \multicolumn{2}{|c|}{ Angle $\left({ }^{\circ}\right)$} & \multirow[b]{2}{*}{$\begin{array}{l}\text { Match the } \\
\text { Algorithm? }\end{array}$} & \multirow[b]{2}{*}{ Comments } \\
\hline & & & & $\begin{array}{l}\text { Proximal } \\
\text { DDR }\end{array}$ & $\begin{array}{l}\text { Distal } \\
\text { DDR }\end{array}$ & $\begin{array}{l}\text { Sup } \\
\text { Angle }\end{array}$ & $\begin{array}{c}\text { Inf } \\
\text { Angle }\end{array}$ & & \\
\hline 1 & 54 & $\mathrm{~F}$ & Rt pterional craniotomy, It OSA aneurysm & 2.5 & 6.2 & 35 & 87 & Yes & $\begin{array}{l}\text { Previous It MCA aneurysm } \\
\text { clipping, rt approach se- } \\
\text { lected to avoid scar tissue }\end{array}$ \\
\hline 2 & 72 & $\mathrm{~F}$ & $\begin{array}{l}\text { Lt mOZ craniotomy, It MCA, It ACoA, \& rt OSA } \\
\text { aneurysm }\end{array}$ & 2.8 & 4.4 & 30 & 75 & Yes & \\
\hline 3 & 53 & M & $\begin{array}{l}\text { Rt pterional craniotomy, rt OSA aneurysm, \& It OSA } \\
\text { aneurysm }\end{array}$ & 2.3 & 5.6 & -30 & 5 & No & $\begin{array}{l}\text { Substantial contralat optic } \\
\text { nerve elevation }\end{array}$ \\
\hline 4 & 79 & $\mathrm{~F}$ & $\begin{array}{l}\text { Rt pterional craniotomy, rt PCoA aneurysm, \& It } \\
\text { OSA aneurysm }\end{array}$ & 3.8 & 5.9 & 21 & 116 & Yes & \\
\hline 5 & 79 & $\mathrm{~F}$ & $\begin{array}{l}\text { Rt mOZ craniotomy, } \mathrm{ACoA} \text { aneurysm, \& It OSA } \\
\text { aneurysm }\end{array}$ & 1.8 & 3.1 & 15 & 35 & No & $\begin{array}{l}\text { Small aneurysm, } 1.3 \mathrm{~mm} \text { in } \\
\text { diameter }\end{array}$ \\
\hline 6 & 23 & M & Rt pterional craniotomy, rt OSA, \& It OSA aneurysm & 2.8 & 5.8 & -31 & 45 & Yes & \\
\hline 7 & 39 & $\mathrm{~F}$ & $\begin{array}{l}\text { Rt pterional craniotomy, rt OSA aneurysm, \& It SHA } \\
\text { aneurysm }\end{array}$ & 3.2 & 6.0 & 40 & 126 & Yes & \\
\hline 8 & 57 & $\mathrm{~F}$ & $\begin{array}{l}\text { Rt pterional craniotomy, rt PCoA, rt MCA, \& It OSA } \\
\text { aneurysm }\end{array}$ & 3.1 & 4.6 & -36 & 33 & Yes & \\
\hline
\end{tabular}

$\mathrm{ACOA}=$ anterior communicating artery; Inf = inferior; $\mathrm{MCA}=$ middle cerebral artery; $\mathrm{mOZ}=$ modified orbitozygomatic; OSA = ophthalmic segment artery; $\mathrm{PCoA}=$ posterior communicating artery; SHA = superior hypophyseal artery; Sup = superior.

contralateral paraclinoid ICA. Because exposure via the interoptic trajectory is limited by bilateral optic nerves, removing the orbit roof does not increase the surgeon's field of vision and is not necessary in this approach. Furthermore, an anterior clinoidectomy, which is needed to obtain proximal control in ipsilateral paraclinoid aneurysms and fully visualize the aneurysm neck, is also not necessary in the contralateral approach.

Adequate exposure of the IOT is associated with elevation of the frontal lobe and requires fully splitting the sylvian fissure and extensively dissecting arachnoid membranes. The olfactory nerve, which connects the basal frontal lobe and anterior skull base, is at risk of damage during the frontal lobe elevation. ${ }^{5,16,17}$ Andrade-Barazarte et al. ${ }^{16}$ reported that the risk of olfactory dysfunction is minimized when the olfactory tract can be fully mobilized after it is dissected free from the surrounding arachnoid adhesions.

\section{Proximal Control}

One of the major concerns associated with the contralateral interoptic trajectory is the difficulty of obtaining proximal control. ${ }^{9,10,18-20}$ Creating room for a temporary clip application proximal to the aneurysm neck that does not obstruct permanent clip reconstruction is optimal; however, this proximal control is rarely feasible because the exposure is limited.

Some authors have exploited cervical ICA balloon occlusion, ${ }^{10,18,20}$ which requires separate interventional equipment. Fries et $a .^{10}$ have also reported incising the DDR after partial tuberculum sellae drilling, which can offer an additional 3-10 $\mathrm{mm}$ of exposure to the clinoid segment ICA. However, this maneuver is invasive and involves the risk of entering the sphenoid sinus.

Transient cardiac arrest with adenosine was advocated by Andrade-Barazarte et al. ${ }^{19,21}$ and has advantages, such as the avoidance of additional cervical incision and the quick recovery of normal circulation. However, the limited time of circulation arrest (20-40 seconds per bolus) may not be sufficient.

Neck dissection with ICA exposure is a common and safe method with respect to proximal control; however, in contralateral approaches, it can be difficult to expose the neck region on the side opposite to the craniotomy. ${ }^{3,10} \mathrm{~A}$ neutral head position allows neck exposure on the contralateral side, and the operating table can be turned as necessary during the operation to achieve both proximal control and appropriate surgical access. ${ }^{22}$

\section{Application of the Proposed Algorithm}

In this study, we proposed a patient-selection algorithm using landmarks that can be identified on both cadaveric specimens and preoperative imaging studies. Our analysis highlights the importance of applying quantitative anatomical studies in clinical neurosurgery. The DDR can be identified as a slightly concave segment on the paraclinoid ICA on a CT angiogram or an angiogram. ${ }^{23}$ Because the position of the DDR is relatively less variable and can be measured in cadavers, the DDR was selected as a landmark. In the clinical validation, 6 of 8 cases were compatible with the algorithm. One of the 2 noncompatible cases involved a small aneurysm (case 5, $1.3 \mathrm{~mm}$ in diameter), and in the other case, substantial retraction to the contralateral optic nerve extended the exposure over the top of the contralateral ICA and to the lateral side (case 3, the inferior neck was only $5^{\circ}$ medially). However, clinical consequences from optic nerve retraction are highly dependent on the tolerance of the optic nerve, which cannot be known intraoperatively and should therefore be avoided. 

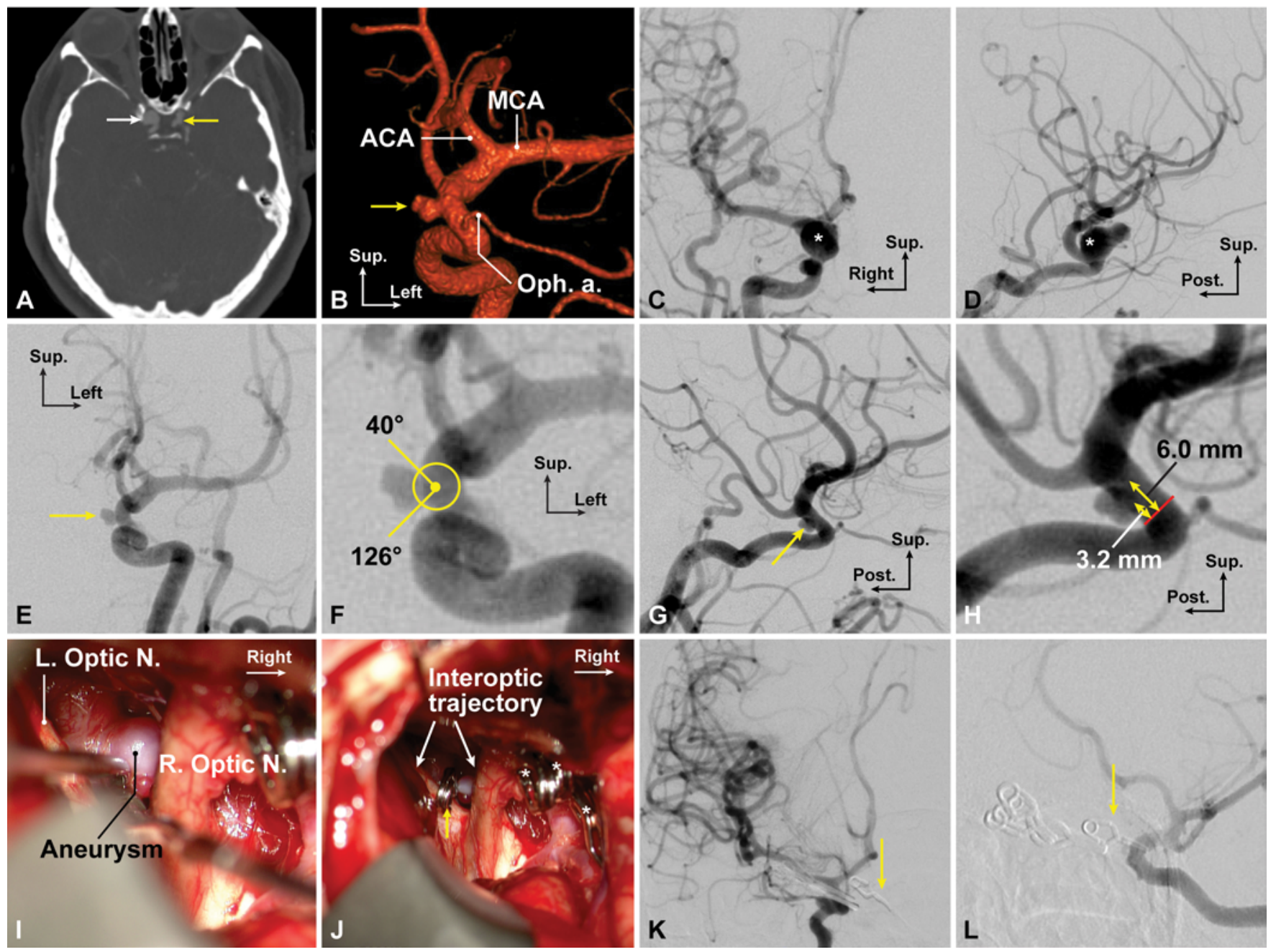

FIG. 5. Case 7. A case example of the application of the algorithm. A 39-year-old woman who presented with syncope and headache was found to have subarachnoid hemorrhage. A: Preoperative CT angiogram showing a right 18-mm supraclinoid aneurysm (white arrow) and a left 4-mm paraclinoid irregular lobulated aneurysm (yellow arrow). B: CT angiogram reconstruction showing the left paraclinoid aneurysm (yellow arrow). C and D: CT angiograms, anteroposterior (C) and lateral (D) views, showing the aneurysm (white asterisks). E and F: CT angiograms, anteroposterior view of left paraclinoid aneurysm (yellow arrow) (E) and magnified detail of this aneurysm $(\mathrm{F})$, which measured $40^{\circ}$ to $126^{\circ}$ on the coronal clockface using the proposed algorithm. $\mathbf{G}$ and $\mathrm{H}$ : CT angiograms, lateral view of left paraclinoid aneurysm (yellow arrow) $(\mathrm{G})$ and magnified detail of this aneurysm $(\mathrm{H})$, which measured 3.2 to $6 \mathrm{~mm}$ (yellow double arrows) to the DDR (red line) using the proposed algorithm. The left paraclinoid aneurysm is compatible with the algorithm. I: A contralateral interoptic approach was selected in a right-to-left direction to access the aneurysm. J: The contralateral aneurysm was successfully clipped (yellow arrow) after the ipsilateral supraclinoid aneurysm was clipped, using a combination of three clips (white asterisks). K: Postoperative angiogram demonstrated complete ligation of the right supraclinoid aneurysm (yellow arrow points to contralaterally placed clip). L: Similarly, postoperative angiogram showed complete ligation of the left paraclinoid aneurysm (yellow arrow points to clip). ACA = anterior cerebral artery; L. Optic N. = left optic nerve; MCA = middle cerebral artery; Oph. $a_{\text {. }}=$ ophthalmic artery; Post. = posterior; R. Optic N. = right optic nerve; Sup. = superior. Published in accordance with the University of California statement on fair use.

Although they did not completely match the algorithm, the measurements of the 2 aneurysms that were successfully clipped via the contralateral interoptic approach were within $1 \mathrm{SD}$ of the mean. The mean values in the algorithm give a range that can be regarded as conservative and safe, and an aneurysm with dimensions that are within this range can be exposed and treated in a relatively straightforward manner with mild or no contralateral optic nerve elevation. An aneurysm with dimensions that are close to but not with- in the range indicated by the algorithm may still be treated with some additional retraction on the contralateral optic nerve; however, the degree of retraction on the contralateral optic nerve must be balanced against the risk of neurological deficits, specifically deterioration in visual acuity or visual field, or even blindness. The algorithm offers general criteria for approach selection; however, selection of this approach should be individualized on the basis of careful and comprehensive evaluation of preoperative imaging. 


\section{Methods to Increase Mobilization of the Contralateral Optic Nerve}

Elevating the contralateral optic nerve can increase exposure to the contralateral paraclinoid ICA because the size of the IOT is relatively limited. Two additional maneuvers to increase mobilization of the contralateral optic nerve have been described in the literature. Incising the contralateral falciform ligament was reported to increase exposure to the paraclinoid ICA by $2 \mathrm{~mm}$ in the proximal direction. ${ }^{5}$ In addition to freeing the falciform ligament, drilling the contralateral portion of the tuberculum sellae and planum sphenoidale increases exposure of the paraclinoid ICA from 6.6 to $9 \mathrm{~mm}^{4}$ These maneuvers increase the interoptic space in a stepwise fashion. ${ }^{7}$

However, drilling the tuberculum sellae and planum sphenoidale can jeopardize the integrity of the sphenoid sinus mucosa and is associated with the risk of cerebrospinal fluid leakage. Moreover, skull base drilling through a narrow and deep trajectory in proximity to crucial neurovascular structures is challenging. In this study, we incised only the contralateral falciform ligament, which is safer and more practical than drilling the tuberculum sellae and planum sphenoidale.

\section{Configuration of the IOT}

Exposure of the contralateral paraclinoid ICA is highly dependent on the size of the IOT, which is defined by the distance between the anterior edge of the chiasm and the limbus sphenoidale (prechiasmatic distance) and the distance between the medial edges of bilateral optic nerves at the entrances of the optic canals (interoptic distance) ${ }^{4,7}$ In a detailed anatomical study, ${ }^{7}$ the size of the IOT expanded after contralateral optic nerve mobilization and contralateral tuberculum sellae and planum sphenoidale debulking. Such maneuvers can increase exposure to the contralateral paraclinoid ICA and may subsequently offer the possibility of proximal control. As discussed above, mobilization of the optic nerve after incising the falciform ligament is considered to be safer than tuberculum sellae debulking. In the literature, it was reported that minimum prechiasmatic distances of $5.4 \mathrm{~mm}^{3}$ and $3.4 \mathrm{~mm}^{19}$ were necessary to obtain sufficient exposure, and minimal sufficient interoptic distances were reported to be at least $10.4 \mathrm{~mm}^{3}$ and $7.6 \mathrm{~mm}^{19}$

A prefixed chiasm is an uncommon phenomenon (found in approximately $3 \%$ of the population) but would be a relative contraindication in the contralateral interoptic approach. ${ }^{24}$ In all cases in our study, the chiasm was positioned normally, but preoperative imaging should be carefully reviewed to rule out a possible prefixed chiasm configuration.

\section{Aneurysm Factors}

If the mass effect of an aneurysm is substantial, the aneurysm neck dissection and exploration can be challenging through this narrow window. Also, the mass effect of an upward-pointing ophthalmic artery aneurysm can impinge on the optic nerve, in which case a full optic canal decompression may be required, which makes the ipsilateral approach more appropriate.
A large and superomedial-pointing aneurysm, which faces and obstructs the direction of the trajectory, may affect direct visualization and exploration of the base of the neck; therefore, proximal control is necessary. Ophthalmic artery aneurysms usually point superiorly, and the superior border of the neck may not be exposed through the IOT. Clip application can still be performed by applying the clip in a medial to lateral direction (Fig. 3A). Similarly, a medially and inferiorly pointing superior hypophyseal artery aneurysm may not have its inferior neck fully exposed in the IOT, and a clip can be applied in a superior to inferior fashion (Fig. 3B). A curved aneurysm clip can be selected, and the curve can be applied along the wall of the carotid artery. In small aneurysms, both borders of which can be exposed through the IOT, an angled clip can be applied (Fig. 3C). As discussed in the proposed algorithm, it is imperative to have at least one of the superior and inferior sides of the aneurysm neck within the range of the exposable angle.

A carotid cave aneurysm is hard to differentiate on the basis of preoperative imaging studies. ${ }^{20}$ Although safe clipping of a carotid cave aneurysm via a contralateral interoptic approach is feasible, ${ }^{20}$ the dome and proximal neck of the aneurysm can hide in the dural pouch between the carotid artery and the sella, ${ }^{25}$ which is unfavorable, and the exposure and dissection to the proximal aneurysm neck can be challenging.

\section{Study Limitations}

The proposed algorithm was based on the measurement of cadavers, the tissue of which is more rigid than live brain parenchyma. Slightly different configurations and sizes of the structures can affect the area exposed. A prefixed optic chiasm is an unfavorable factor for a contralateral interoptic approach to a paraclinoid aneurysm. Prefixed chiasms are rare and were not encountered in our cadaveric study. When considering this approach clinically, preoperative imaging should be carefully reviewed to exclude a prefixed chiasm. The superior border of the exposable area is modified by the extent of contralateral optic nerve elevation. This maneuver increases the area of the IOT but may have consequences for optic nerve function. The tolerance of the optic nerve to this maneuver is difficult to determine in clinical practice and is impossible to examine in the laboratory setting.

\section{Conclusions}

The contralateral interoptic approach is a safe and feasible route to access aneurysms that arise from the medial paraclinoid ICA. An aneurysm can be safely treated via the contralateral interoptic trajectory if 1) both proximal and distal borders of the aneurysm neck are $2.5-8.4 \mathrm{~mm}$ distal to the DDR, and 2) at least one border of the aneurysm neck on the coronal clockface is $21.7^{\circ}-130.9^{\circ}$ medial to the vertical line.

\section{Acknowledgments}

We thank the staff of Neuroscience Publications at Barrow Neurological Institute for assistance with manuscript and illustration preparation. 


\section{References}

1. Day AL. Aneurysms of the ophthalmic segment. A clinical and anatomical analysis. J Neurosurg. 1990;72(5):677-691.

2. Serrano LE, Ayyad A, Archavlis E, et al. A literature review concerning contralateral approaches to paraclinoid internal carotid artery aneurysms. Neurosurg Rev. 2019;42(4):877884.

3. Kakizawa Y, Tanaka Y, Orz Y, et al. Parameters for contralateral approach to ophthalmic segment aneurysms of the internal carotid artery. Neurosurgery. 2000;47(5):1130-1137.

4. Serrano LE, Archavlis E, Ayyad A, et al. Comprehensive anatomic assessment of ipsilateral pterional versus contralateral subfrontal approaches to the internal carotid ophthalmic segment: a cadaveric study and three-dimensional simulation. World Neurosurg. 2019;128:e261-e275.

5. Yu LH, Shang-Guan HC, Chen GR, et al. Monolateral pterional keyhole approaches to bilateral cerebral aneurysms: anatomy and clinical application. World Neurosurg. 2017;108:572-580.

6. Sheikh B, Ohata K, El-Naggar A, et al. Contralateral approach to junctional $\mathrm{C} 2-\mathrm{C} 3$ and proximal $\mathrm{C} 4$ aneurysms of the internal carotid artery: microsurgical anatomic study. Neurosurgery. 2000;46(5):1156-1161.

7. Serrano LE, Archavlis E, Ayyad A, et al. The approach angle to the interoptic triangle limits surgical workspace when targeting the contralateral internal carotid artery. Acta Neurochir (Wien). 2019;161(8):1535-1543.

8. He H, Chen C, Li W, et al. Contralateral approach based on a preoperative 3-dimensional virtual osteotomy technique for anterior circulation aneurysms. J Stroke Cerebrovasc Dis. 2019;28(4):1099-1106.

9. Wang J, Wu J, Cao Y, et al. Contralateral approach to paraclinoid aneurysms: angiographic analysis and surgical results of 12 patients. J Neurol Surg A Cent Eur Neurosurg. 2019;80(3):180-186.

10. Fries G, Perneczky A, van Lindert E, Bahadori-Mortasawi F. Contralateral and ipsilateral microsurgical approaches to carotid-ophthalmic aneurysms. Neurosurgery. 1997;41(2):333343.

11. Loumiotis I, D’Urso PI, Tawk R, et al. Endovascular treatment of ruptured paraclinoid aneurysms: results, complications, and follow-up. AJNR Am J Neuroradiol. 2012;33(4):632-637.

12. Chen Z, Yang Y, Miao H, et al. Experiences and complications in endovascular treatment of paraclinoid aneurysms. $J$ Clin Neurosci. 2013;20(9):1259-1263.

13. Thornton J, Aletich VA, Debrun GM, et al. Endovascular treatment of paraclinoid aneurysms. Surg Neurol. 2000;54(4):288-299.

14. Shimizu K, Imamura H, Mineharu Y, et al. Endovascular treatment of unruptured paraclinoid aneurysms: single-center experience with 400 cases and literature review. AJNR Am J Neuroradiol. 2016;37(4):679-685.

15. Krisht AF, Hsu SP. Paraclinoid aneurysms: Part 1: Superior (true ophthalmic) aneurysms. Contemp Neurosurg. 2008;30(15):1-5.

16. Andrade-Barazarte H, Kivelev J, Goehre F, et al. Contralateral approach to bilateral middle cerebral artery aneurysms: comparative study, angiographic analysis, and surgical results. Neurosurgery. 2015;77(6):916-926.

17. Park J, Lee SH, Kang DH, Kim JS. Olfactory dysfunction after ipsilateral and contralateral pterional approaches for cerebral aneurysms. Neurosurgery. 2009;65(4):727-732.

18. Chen S, Kato Y, Kumar A, et al. Contralateral approach to unruptured superior hypophyseal artery aneurysms. J Neurol Surg A Cent Eur Neurosurg. 2013;74(1):18-24.

19. Andrade-Barazarte H, Kivelev J, Goehre F, et al. Contralateral approach to internal carotid artery ophthalmic segment aneurysms: angiographic analysis and surgical results for 30 patients. Neurosurgery. 2015;77(1):104-112.

20. Sheikh B, Ohata K, El-Naggar A, et al. Contralateral approach to carotid cave aneurysms. Acta Neurochir (Wien). 2000;142(1):33-37.

21. Andrade-Barazarte H, Luostarinen T, Goehre F, et al. Transient cardiac arrest induced by adenosine: a tool for contralateral clipping of internal carotid artery-ophthalmic segment aneurysms. World Neurosurg. 2015;84(6):1933-1940.

22. Chandela S, Chakraborty S, Ghobrial GM, et al. Contralateral mini-craniotomy for clipping of bilateral ophthalmic artery aneurysms using unilateral proximal carotid control and Sugita head frame. World Neurosurg. 2011;75(1):78-82, 41-42.

23. Murayama Y, Sakurama K, Satoh K, Nagahiro S. Identification of the carotid artery dural ring by using three-dimensional computerized tomography angiography. Technical note. J Neurosurg. 2001;95(3):533-536.

24. Gulsen S, Dinc AH, Unal M, et al. Characterization of the anatomic location of the pituitary stalk and its relationship to the dorsum sellae, tuberculum sellae and chiasmatic cistern. $J$ Korean Neurosurg Soc. 2010;47(3):169-173.

25. Hitotsumatsu T, Natori Y, Matsushima T, et al. Microanatomical study of the carotid cave. Acta Neurochir (Wien). 1997;139(9):869-874.

\section{Disclosures}

The authors report no conflict of interest concerning the materials or methods used in this study or the findings specified in this paper.

\section{Author Contributions}

Conception and design: Lawton, Zhao. Acquisition of data: Zhao, Labib, Naeem. Analysis and interpretation of data: Zhao, Gandhi, Belykh. Drafting the article: Zhao, Tayebi Meybodi. Critically revising the article: Lawton, Tayebi Meybodi, Labib, Gandhi, Belykh, Naeem, Preul, Nakaji. Reviewed submitted version of manuscript: Lawton. Statistical analysis: Zhao. Administrative/ technical/material support: Lawton, Preul, Nakaji. Study supervision: Lawton.

\section{Correspondence}

Michael T. Lawton: c/o Neuroscience Publications, Barrow Neurological Institute, St. Joseph's Hospital and Medical Center, Phoenix, AZ.neuropub@barrowneuro.org. 